\title{
Audio Watermarking Scheme Robust against Desynchronization Based on the Dyadic Wavelet Transform
}

\author{
Yong Wang, Shaoquan Wu, and Jiwu Huang \\ Guangdong Province Key Laboratory of Information Security, School of Information Science and Technology, Sun Yat-Sen University, \\ Guangzhou, Guangdong, China
}

Correspondence should be addressed to Jiwu Huang, isshjw@mail.sysu.edu.cn

Received 6 April 2009; Revised 3 September 2009; Accepted 12 January 2010

Academic Editor: Aggelos Pikrakis

Copyright ( 2010 Yong Wang et al. This is an open access article distributed under the Creative Commons Attribution License, which permits unrestricted use, distribution, and reproduction in any medium, provided the original work is properly cited.

Digital watermarking is a technique used to embed an extra piece of information into multimedia signals without degrading the signal quality. For robust audio watermarking, geometrical modifications are common operations and present many challenges because they severely alter the tempo or spectral structures of the audio and thus cause watermark desynchronization. However, most of the existing audio watermarking algorithms lack resynchronization ability due to the nongeometrically-invariant nature of the watermarking domain. In this paper, we consider the dyadic wavelet transform (DYWT) for its geometrical invariants which can help resynchronize the watermark. We then design a novel embedding method based on shape modulation which is demonstrated to be robust against many kinds of attack. Based on the knowledge of the insertion, deletion, and substitution (IDS) channel, we carefully design a novel error correction coding (ECC) with the ability of bit-resynchronization to correct the IDS errors in the watermark. Compared with existing algorithms, our algorithm achieves greater robustness to geometrical modifications and other common operations.

\section{Introduction}

Any operation that modifies the audio signal in the time domain or transform domain may result in loss or change of the watermark that is hidden in the audio. Therefore, the watermark algorithm must be able to recognize the parts that contain the watermark, to recover the lost hidden bits and to remove the added extra bits from the watermark.

For example, geometrical transforms, such as time scale modification (TSM) and pitch scale modification (PSM), are common operations on audio signals. According to [1, 2], they are common attacks that a copyright watermark must withstand because they can seriously damage the synchronization of the watermark. Compared with other magnitude-distortions caused by attacks or operations such as noise, compression, low-frequency filtering, resampling, requantization, and so forth, desynchronization caused by geometrical modifications are the most difficult problems to overcome in audio watermarking.

Many efforts in image watermarking that are robust to desynchronization caused by geometrical distortions have been reported [3-9]. In audio watermarking, some works have tried to find ways that can resist desynchronization. In our previous work [10], we realized the problem of synchronization and embedded Bark codes into the time domain to indicate the segments on which the discrete cosine transform (DCT) should be performed. But the Bark codes are easy to erase if subjected to TSM or PSM. Mansour and Tewik [11] proposed a watermarking scheme by quantizing the distances between the peaks of the low-frequency region. This scheme is reported to be robust to $\pm 2 \%$ TSM. He also embedded the data by modifying the ratio of intervals between successive maxima and minima pairs at a rate of $1 \sim 2$ bps (bits per second) of hidden bits [12]. However, according to [13], the watermark should be robust to a TSM of $\pm 10 \%$. $[11,12]$ cannot meet this requirement. In [14], Wang et al. also proposed a DYWT-based algorithm which is unable to resist PSM. Li et al. $[15,16]$ proposed algorithms that used the peaks of the drum-frequency band of a piece of music for synchronizing the watermark embedding-regions, which is very robust to strong geometrical attacks, for example, TSM up to $\pm 18 \%$, but it is not robust to PSM. Cui et al. 
[17] supposed the complex cepstrum a good domain for embedding which could withstand geometrical distortions. However neither the corresponding theoretical analysis nor experimental results were given in the paper. The authors in [18] claimed that their algorithm based on the discrete wavelet transform (DWT) could withstand TSM without reporting experimental results. In reference [19], the audio signal was first divided into several frames of the same length and the watermark bits were embedded into the frames, which could withstand $\pm 3 \%$ TSM. Xiang and Huang [20] proposed a histogram-based algorithm that can resist $-20 \% \sim+30 \%$ TSM. Wang et al. [21] resynchronized the extraction process by adopting an adaptive segmentation step. But it only solves desynchronization caused by some MP3 encoders (an extra segment of around 1000 samples added by encoders to change the length of the audio). When more complicated modifications occur, adaptive segmentation would become ineffective. Liu et al. [22] also paid attention to desynchronization but did not propose a way to solve it. None of the above algorithms can resist PSM. Li et al. [23] proposed a spread-spectrum and one-bit algorithm against PSM. But it is unable to resist TSM.

Briefly, the main problem of existing reports is the lack of an effective way to resist both TSM and PSM, along with other modifications such as cropping, jittering, compression, resampling, and so forth. Domains such as DFT or DCT, which are employed in $[10,15,16]$ do not have invariant properties under both TSM and PSM. DFT, DCT and DWT also have drawbacks in resistance to time shifting, which is the main problem of $[15,16,19]$. The method for extracting the watermark is also delicate. If it depends on the precise number of participated samples for extraction, it will probably fail if the audio has been processed by TSM, PSM or cropping, because after such modifications, the number of samples in the time or frequency domain will change [2023] all exhibit this problem.

Aiming to solve the above problems, we propose in this paper an audio watermarking algorithm based on DYWT which is robust to both TSM and PSM and which utilizes the geometrical invariance of DYWT for watermark resynchronization. A well-designed ECC based on repetition coding is integrated into the algorithm for watermark selfsynchronization. The algorithm is also robust against cropping, jittering, and most of the attacks of Stirmark, which means that it is robust against most common operations.

The structure of this paper is as follows. We prove the geometrical invariance of DYWT in Section 2. The watermarking scheme is given in Section 3. The experimental results and comparison with other reported work are presented in Section 4. Finally, we summarize the conclusions and discuss some related issues and future work in Section 5.

\section{Geometrical Invariance of DYWT}

Although DFT, DCT and especially DWT are applied for audio watermarking widely, they have drawbacks in terms of geometrical invariance. Let us give a brief discussion.

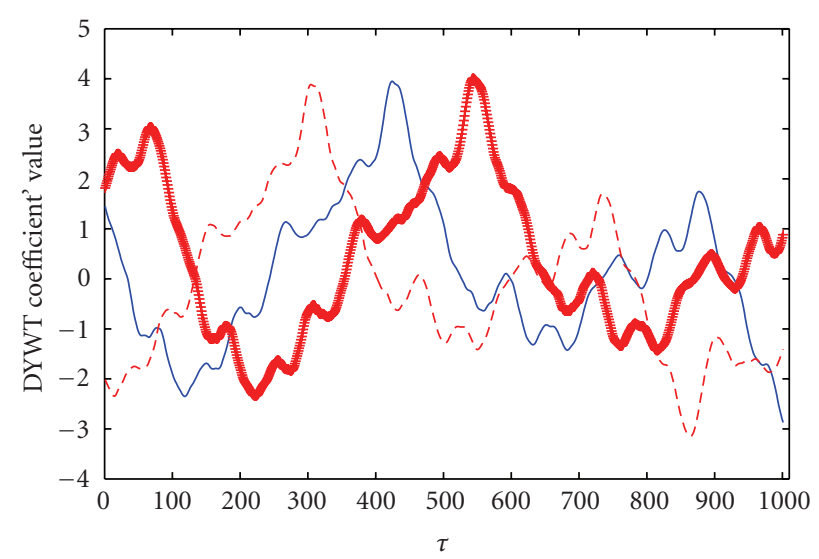

(a) Temporally linear TSM

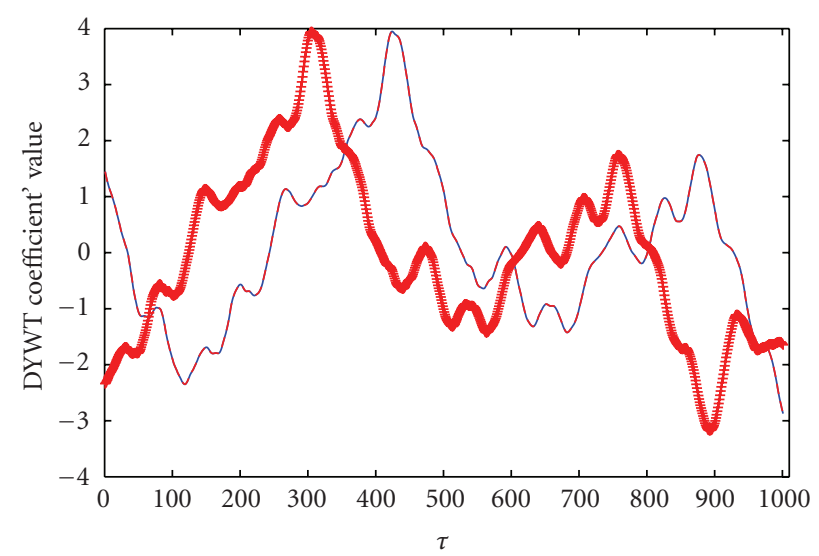

(b) Pitch invariant TSM

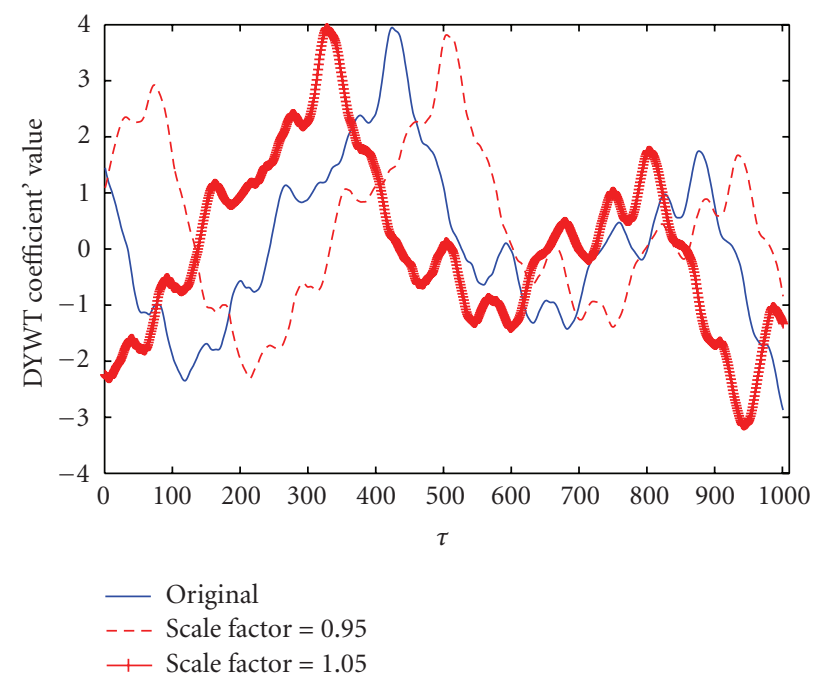

(c) Temporally invariant PSM

FIGURE 1: Invariance of DYWT to TSM and PSM.

In [24], the relationship between the two-filter-DWT coefficients and the time shift is deduced. In this paper, we outline a more general conclusion about this relationship in Appendix A. From Appendix A, we know that when a signal is shifted $N$ positions in the time domain and $N=n \cdot 2^{j}$, the $j$ th level DWT coefficients will be shifted $n$ positions 


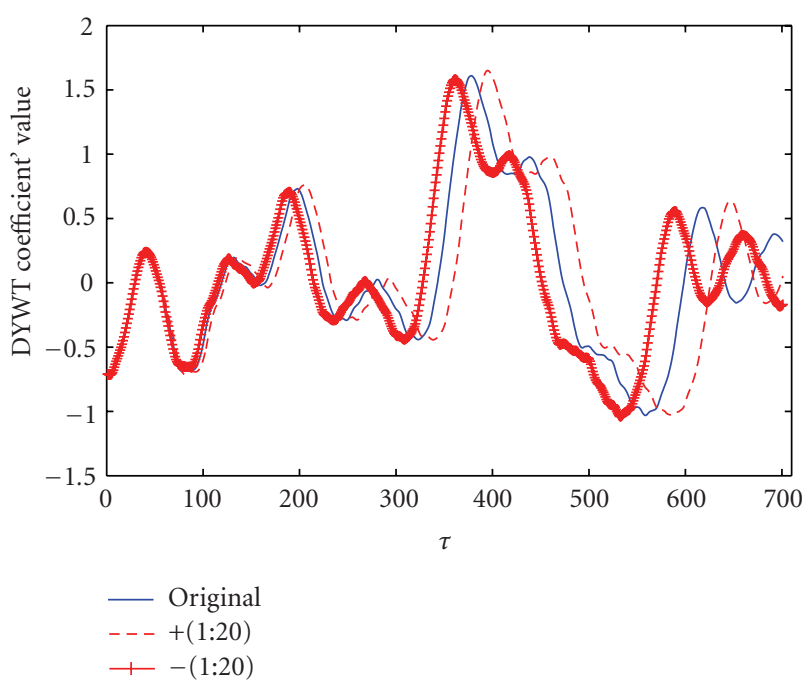

FIGURE 2: Invariance of DYWT to jittering.

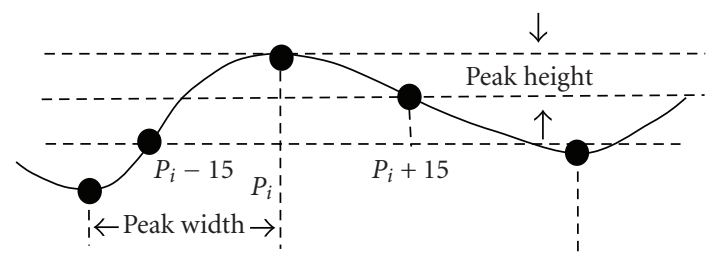

FIgURE 3: Peak width and peak height.

along the same direction. Under this condition, the DWT has the property of time shift invariance. However condition (A.4) (conditions (A.6), (A.7)) in Appendix A does not hold when $j$ increases beyond a certain number and $N$ is not a multiple of of $2^{j}$. At that time, the DWT does not have the property of shift invariance. For example, if the watermark is embedded into the 5th level of decomposition, $N$ should be $0,32,64, \ldots$, in order to have identical DWT coefficients. However, it is uncertain how many positions a signal will be shifted when attacked. On the other hand, when DFT or DCT is applied to audio watermarking it must be emphasized that the audio signal is always divided into segments, and it is on these segments that the DFT or DCT is performed. Time shifting and other geometrical modifications will always lead to the incorrect identification of the segment boundaries. Generally $\operatorname{DFT}(S) \neq \operatorname{DFT}\left(S^{\prime}\right)$ and $\operatorname{DCT}(S) \neq \operatorname{DCT}\left(S^{\prime}\right)$ when $S$ and $S^{\prime}$ are not aligned. So neither the DFT nor the DCT are time-shift invariant. These are the limitations of DWT, DFT and DCT in the application of audio watermarking.

Based on the above knowledge, we began to investigate the properties of other transforms and found that the DYWT has invariant features to geometrical modifications that can be used for resynchronization. In this section we examine the properties of the DYWT by theoretical analysis and in extensive experiments.
According to wavelet theory, the DYWT is discretized along the vertical scale axis but is continuous along the time axis. The dyadic wavelet can be expressed as

$$
\psi_{2^{k}, \tau}(t)=2^{-k / 2} \psi\left(\frac{t-\tau}{2^{k}}\right)
$$

Suppose $\mathrm{WT}_{2^{k}}(\tau)$ is the $k$ th level DYWT coefficient of $f(t)$. Then

$$
\mathrm{WT}_{2^{k}}(\tau)=f(t)^{*} \psi_{2^{i}, \tau}(t)=2^{-k / 2} \int_{R} f(t) \psi\left(\frac{\tau-t}{2^{k}}\right) d t .
$$

2.1. Invariance to Time Shifting. The DYWT of $f\left(t-\tau_{0}\right)$ and $f(\beta \cdot t)$ can be represented as

$$
\begin{aligned}
\mathrm{WT}_{2^{k}}^{\prime}(\tau) & =f\left(t-\tau_{0}\right)^{*} \psi_{2^{k}, \tau}(t) \\
& =2^{-k / 2} \int_{R} f\left(t-\tau_{0}\right) \psi\left(\frac{\tau-t}{2^{k}}\right) d t \\
& =2^{-k / 2} \int_{R} f\left(t^{\prime}\right) \psi\left(\frac{\tau-\tau_{0-} t^{\prime}}{2^{k}}\right) d t^{\prime} \\
& =2^{-k / 2} \int_{R} f(t) \psi\left(\frac{\tau-\tau_{0-}}{2^{k}}\right) d t \\
& =\mathrm{WT}_{2^{k}}\left(\tau-\tau_{0}\right) .
\end{aligned}
$$

It can be shown from (3) that DYWT is invariant with shifts in the time domain. That is, if the audio signal is shifted in the time domain, its DYWT coefficients will be shifted identically without any changes.

2.2. Invariance to TSM and PSM. TSM and PSM have wide applications in the audio community such as synthesis by resampling, post-synchronization, data compression, reading for the blind, foreign language learning, computer interface, post-production sound editing, musical composition, and so forth [25]. Temporal linear scaling stretches an audio signal with both duration and pitch changes. Pitch invariant TSM modifies the duration of a signal without altering its pitch while PSM modifies the pitch of a signal without changing its duration. In this section we prove that the DYWT is approximately invariant to both TSM and PSM.

Given that the temporal linear scaling factor is $\beta$, the DYWT of $f(\beta \cdot t)$ can be represented as

$$
\begin{aligned}
\mathrm{WT}_{2^{k}}^{\prime \prime}(\tau) & =f(\beta \cdot t)^{*} \psi_{2^{k}, \tau}(t) \\
& =2^{-k / 2} \int_{R} f(\beta \cdot t) \psi\left(\frac{\tau-t}{2^{k}}\right) d t \\
& =\frac{2^{-k / 2}}{\beta} \int_{R} f\left(t^{\prime}\right) \psi\left(\frac{\tau-\left(t^{\prime} / \beta\right)}{2^{k}}\right) d t^{\prime} \\
& =\frac{2^{-k / 2}}{\beta} \int_{R} f(t) \psi\left(\frac{\beta \tau-t}{\beta 2^{k}}\right) d t .
\end{aligned}
$$

From (4) we can show that, if

$$
\beta=2^{m}, \quad m \in Z,
$$



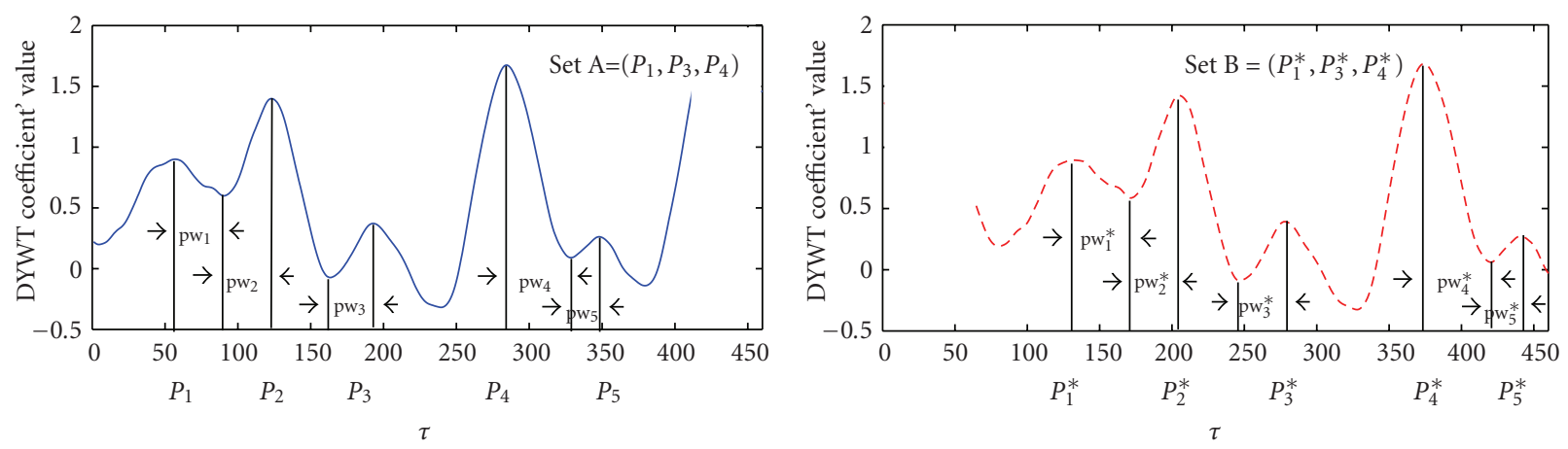

FIGURE 4: The widest peaks used for embedding and extracting watermark bits.

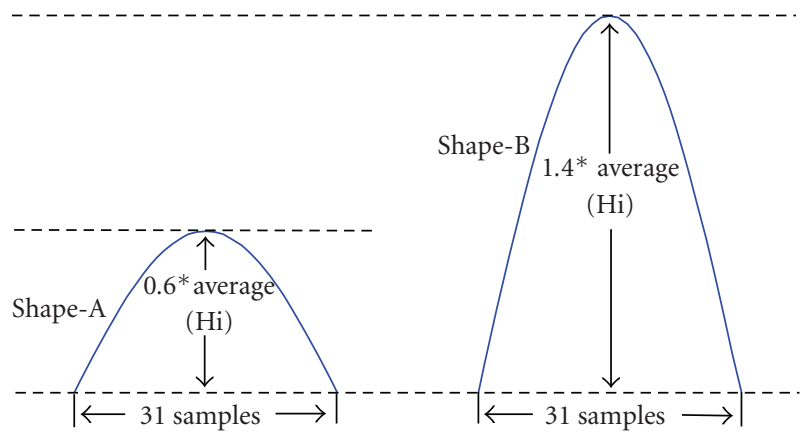

Figure 5: Shape-A and Shape-B.

then

$$
\mathrm{WT}^{\prime \prime}{ }_{2^{k}}(\tau)=\frac{1}{\beta} \cdot \mathrm{WT}_{2^{k+m}}(\beta \cdot \tau)
$$

At this moment, the DYWT is temporal liner scaling invariant because the DYWT of the signals can be obtained from the original DYWT as they are different only by a scale factor $\beta$. Certainly, generally $\beta \neq 2^{m} m \in Z$ and $k+\log _{2} \beta$ is not a decomposition level that can be reached. Nevertheless, the DYWT is scaling invariant to some extent. Let us examine this now.

In most practical applications, $0.8 \leq \beta \leq 1.2$. Then $k-$ $0.3219 \leq k+\log _{2} \beta \leq k+0.2630$, and floor $\left(k+\log _{2} \beta+0.5\right)=k$. Thus we have

$$
\begin{aligned}
\mathrm{WT}_{2^{k}}^{\prime \prime}(\tau) & =\frac{2^{-k / 2}}{\beta} \int_{R} f(t) \psi\left(\frac{\beta \tau-t}{\beta 2^{k}}\right) d t \\
& \approx \frac{2^{-k / 2}}{\beta} \int_{R} f(t) \psi\left(\frac{\beta \tau-t}{2^{k}}\right) d t \\
& =\frac{1}{\beta} \mathrm{WT}_{2^{k}}(\beta \tau) .
\end{aligned}
$$

Equation (7) shows that the DYWT is approximately scale invariant along scale $k$, which is verified by extensive experiments.

Since there are different implementations of pitch invariant TSM and temporal invariant PSM, it is hard to give an explicit mathematical relationship like (7) for these two kinds of scaling. According to [26], a signal can be represented as a sum of sinusoids whose instantaneous frequency and instantaneous amplitude vary slowly with time. Ideal pitch invariant TSM corresponds to moving the instantaneous amplitudes of the sinusoids from $t$ to $\beta t$ with unchanged instantaneous frequencies and changed instantaneous phases. The modification of the amplitudes is similar to temporal linear TSM [25]. So, we believe that the DYWT is invariant to this TSM to some extent. Also, since temporal invariant PSM can be obtained by a temporally linear TSM and pitch invariant TSM, we also expect DYWT to have the same property under PSM. Our beliefs have been confirmed by extensive experiments. Here, we show some experimental results in Figure 1, in which the coefficients of the DYWT low frequency subband of an audio clip (symphony) and its TSM and PSM versions are shown. The wavelet basis is $\mathrm{db} 2$ and the decomposition level is 5. It can be observed that the DYWT is to a large extent invariant to both TSM and PSM. That is, the shapes of the waveforms remain approximately unchanged after TSM or PSM. Therefore, if features such as local maxima, local minima or the fast energy transitions are used for synchronizing or embedding the watermark bits, the watermark promises to withstand relatively strong TSM and PSM attacks.

2.3. Invariance to Jittering and Cropping. Jittering is the deletion/insertion of samples evenly throughout a signal. $+(1: \mathrm{N})$ refers to copying one sample into each segment of $\mathrm{N}$ samples; $-(1: \mathrm{N})$ refers to deleting one sample from each segment of $\mathrm{N}$ samples. The invariance of DYWT to jittering is also verified by experiments, with an example shown in Figure 2. We can see that after jittering, the waveform of the DYWT coefficients remains similar to the original one. Cropping refers to cutting off of some portion of an audio signal. When a portion is cropped, those watermark bits in that portion will be lost. But because DYWT is invariant against time shifts, other watermark bits can still be retained in the remaining parts. Further countermeasures must be taken in order to prevent error propagation and to recover the original watermark due to the lost bits, as will be introduced in Section 3. 


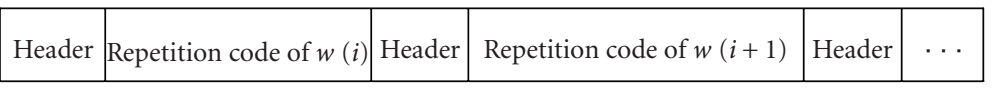

FIGURE 6: Watermark structure after RHC.

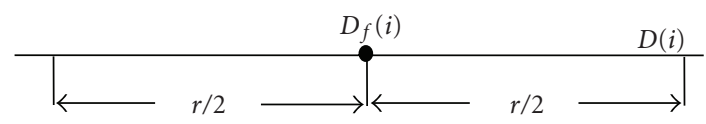

FIgURE 7: The operation of bit filtering.
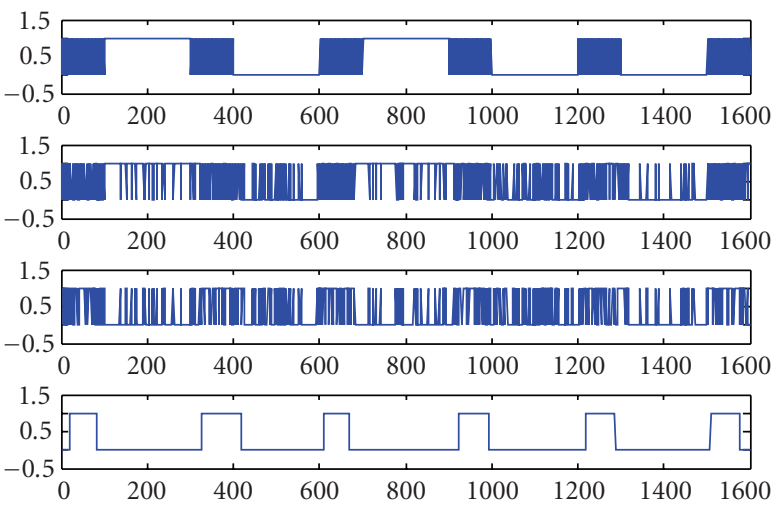

Figure 8 : The 1 st to 4 th figures are $w_{1}, w_{1}^{*}, D$, and $D_{f}$, respectively.

Briefly, we arrive at the conclusion that the DYWT has very good geometrically invariant properties, which makes it an appropriate carrier for a watermark.

2.4. Comments on the Invariant Features of DYWT and Resynchronization of Watermark. The watermark extractor must identify the portions that contain the watermark bits before extracting them. This identification is called watermark resynchronization. It can be achieved by two main schemes, template matching [10] or the employment of invariant features $[11,12,14-16,20]$. The former scheme is fragile because the template itself is easy to destroy. In the latter scheme, the watermark is embedded into the invariant features of the cover signal. It is more robust than the former if the selected features are robust enough. Since the relation features of DYWT remain invariant to various modifications as stated in the above section, we adopt the latter scheme.

Through extensive experiments, we find that the relation between peak widths is extremely robust to various modifications. Therefore, in this paper, the widest peaks of the DYWT low frequency sub-band are selected to contain the watermark bits. The peak width (pw) is defined as the minimum of the two distances between the peak point and its right-sided and left-sided troughs, as illustrated in Figure 3.

Suppose $L$ bits are to be embedded. Then select $L$ peak points with the widest peak width for the watermark bit embedding. In Figure 4, we show a portion of the 5 level DYWT low frequency sub-band of an audio signal. Suppose the watermark contains 3 bits and $\mathrm{pw}_{4}>\mathrm{pw}_{1}>\mathrm{pw}_{3}>\mathrm{pw}_{2}>$ $\mathrm{pw}_{5}$. Then we select $P_{1}, P_{3}$ and $P_{4}$ for bit embedding. After scaling, we can see that due to the time shift and scaling invariance of the DYWT, the relationship between the peak widths still remains: $\mathrm{pw}_{4}^{*}>\mathrm{pw}_{1}^{*}>\mathrm{pw}_{3}^{*}>\mathrm{pw}_{2}^{*}>\mathrm{pw}_{5}^{*}$ as shown in Figure 4. Thus we can extract the watermark bits from $P_{1}^{*}, P_{3}^{*}$, and $P_{4}^{*}$.

For clarity, the set of peaks that are selected for bit embedding is called Set A; and the set of peaks that are considered as containing the watermark bits in the extraction process is called Set B. Apparently the degree of similarity between these two sets reflects the invariance features of the DYWT and is a key factor for resynchronization. In Section 4 we will analyse this similarity by experiment and prove that this feature is very robust against various kinds of modifications.

\section{Proposed Watermarking Scheme}

3.1. Data Embedding. For greater robustness, data embedding should not rely on any particular DYWT coefficient because the values of the coefficients always change during the transmission. A good method for embedding is to use a certain length of the waveform to represent the watermark bits. Also, since we have selected peak widths as the resynchronization criteria, we should not change the peak widths during data embedding. Here, we construct two different waveforms, Shape-A and Shape-B, to represent "0" and "1", respectively, as shown in Figure 5.

The details are as follows.

(1) Perform $K$-level DYWT decomposition on the audio signal. The low frequency sub-band, denoted by $\mathrm{WT}_{2^{k}}(\tau)$, is used to contain the watermark $w_{1}$.

(2) Denote all the peaks in $\mathrm{WT}_{2^{k}}(\tau)$ as $\left\{P_{i}\right\}$. Calculate the height of every peak. A peak height is defined as the difference in heights between $P_{i}$ and $P_{i}+15$, and between $P_{i}$ and $P_{i}-15$, as illustrated in Figure 3 . Denote the heights of all peaks $\left\{P_{i}\right\}$ as $\left\{H_{i}\right\}$.

(3) Construct two waveforms, Shape-A and Shape-B, as shown in Figure 5.

(i) Shape-A $=0.6^{*}$ average $\left\{H_{i}\right\} * \sin ([0: \pi / 30$ : $\pi]$ )

(ii) Shape- $\mathrm{B}=1.4^{*}$ average $\left\{H_{i}\right\} * \sin ([0: \pi / 30$ : $\pi]$ )

(4) Modulate the shape of the waveform between $P_{\mathrm{i}}-15$ and $P_{\mathrm{i}}+15\left(P_{i} \in S E T \mathrm{~A}\right)$ according to the following rules.

(i) If $w_{1}(j)=0$ and $H_{i}<1.4 \times$ average $\left\{H_{i}\right\}$, then the original shape is replaced by Shape- $\mathrm{B}$, that is, $\mathrm{WT}_{2^{k}}\left(P_{i}-15: P_{i}+15\right)=$ Shape-B. 


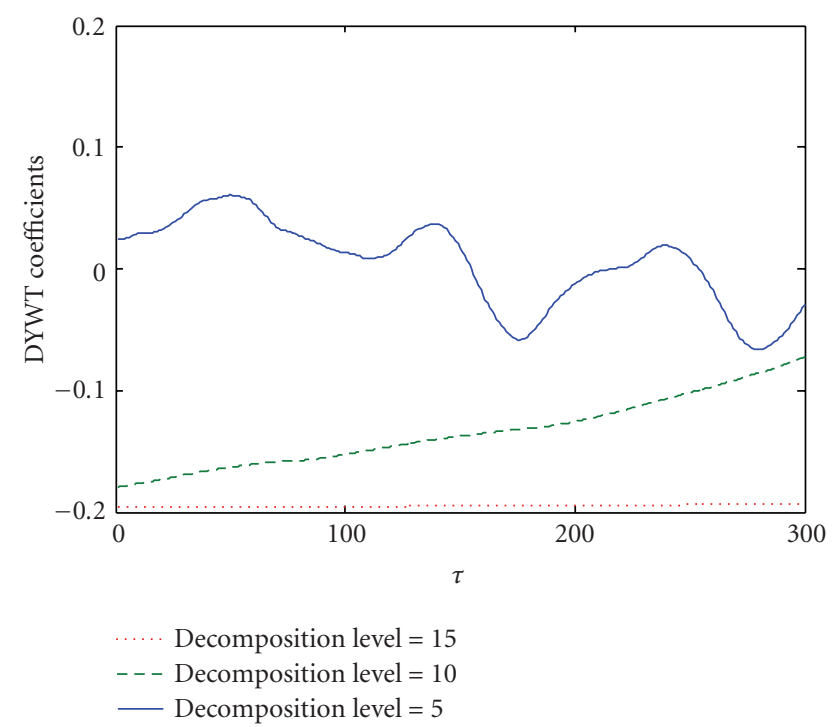

(a)

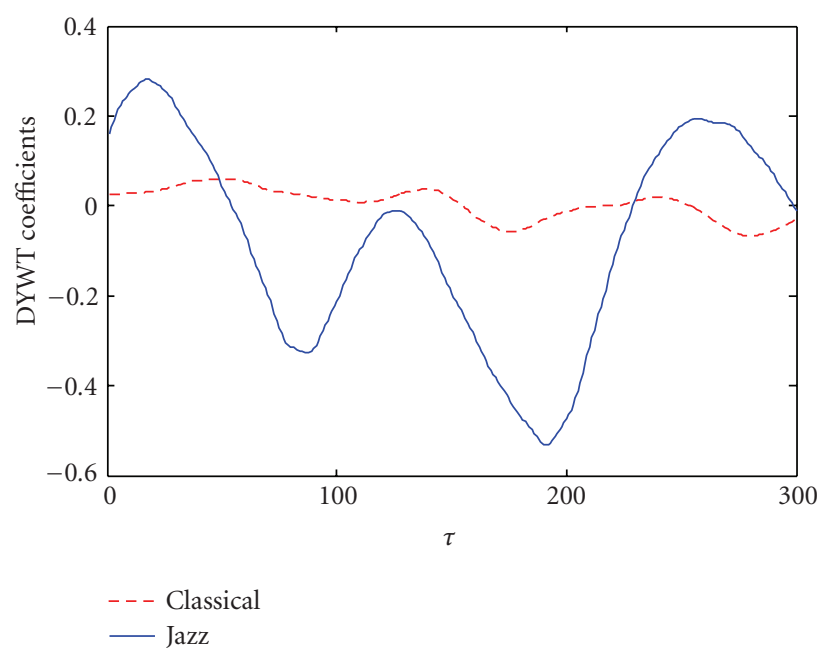

(b)

FIGURE 9: (a) Fluctuation comparison between DYWT coefficients of different decomposition level, (b) fluctuation comparison between DYWT coefficients of different clips under the same decomposition level.

(ii) If $w_{1}(j)=1$ and $H_{i}>0.6 \times$ average $\left\{H_{i}\right\}$, then the original shape is replaced by Shape-A, that is, $\mathrm{WT}_{2^{k}}\left(P_{i}-15: P_{i}+15\right)=$ Shape-A.

(iii) Otherwise no modification is needed.

(5) Perform the inverse DYWT and obtain the watermarked audio signal. Since the DYWT is not a nonredundancy transform 1 , the modifications made in the $K$-level DYWT subband may not be completely reserved after reconstruction. Therefore a loop is needed, performing a K-level DYWT decomposition on the modified audio signal and checking to see whether the shape of its low frequency sub-band satisfies the requirements of the rules in step (4). If all the shapes need not to be modified anymore, this audio clip is the final watermarked clip. Otherwise return to step (4) and continue the modulation. Eventually, we will obtain the watermarked signal.

The watermark extraction process includes the following steps.

(1) Perform K-level DYWT decomposition. In the low frequency sub-band, select those peaks with the widest width as the elements of Set $B$. Denote all the peaks in the sub-band as $\left\{P_{i}^{*}\right\}$. Denote the heights of all peaks $\left\{P_{i}^{*}\right\}$ as $\left\{H_{i}^{*}\right\}$.

(2) Suppose $P_{i}^{*}$ is the $j$ th element in Set B. The decision is made according to the height of $P_{i}^{*}$ :

$$
w_{1}^{*}(j)= \begin{cases}0, & \text { if } H_{i}^{*}>\text { average }\left\{H_{i}^{*}\right\}, \\ 1, & \text { otherwise. }\end{cases}
$$

According to wavelet theory, the frequency range of the Kth DYWT low frequency sub-band is $\left[0, F / 2^{K+1}\right]$, where $\mathrm{F}$ is the sampling frequency. Compared with the frequency range of most musical instruments [27], $K$ can be chosen to be 3 , 4, or 5 when $F=44100 \mathrm{~Hz}$.

3.2. Desynchronization Attack Channel and ECC. Let us review Figure 4. If no modification is performed on the watermarked signal $f^{\prime}$ or the modification is not strong enough, the $L$ widest peaks (Set A) at the embedding end will remain as the $L$ widest peaks (Set B) at the extraction end, as shown in Figure 4. Then all the watermark bits will be extracted from the correct positions. No bit desynchronization occurs in this situation. But if the modification is strong enough, the relation between the peak widths may change and thus Set B may differ from Set A. For example, in Figure 4 let us suppose that after modification, the relation of the peak widths becomes $\mathrm{pw}_{4}^{*}>\mathrm{pw}_{1}^{*}>$ $\mathrm{pw}_{5}^{*}>\mathrm{pw}_{3}^{*}>\mathrm{pw}_{2}^{*}$. Then we will extract the watermark bits from Set $\mathrm{B}=\left\{P_{1}^{*}, P_{4}^{*}, P_{5}^{*}\right\}$ instead of $\left\{P_{1}^{*}, P_{3}^{*}, P_{4}^{*}\right\}$. We can see that the watermark bit contained in $P_{3}^{*}$ is lost (deleted), and an extra bit extracted from the unwatermarked $P_{5}^{*}$ is inserted after the bit is extracted from $P_{4}^{*}$. These two kinds of errors will cause the watermark bits to be shifted forward or backward, and thus corrupt the synchronization of the watermark bits. Even from a truly watermarked peak, a wrong bit may be extracted due to the changed values of samples, which is called a substitution error. For watermarking, all attack channels can be viewed as insertion, deletion and substitution (IDS) channels. 

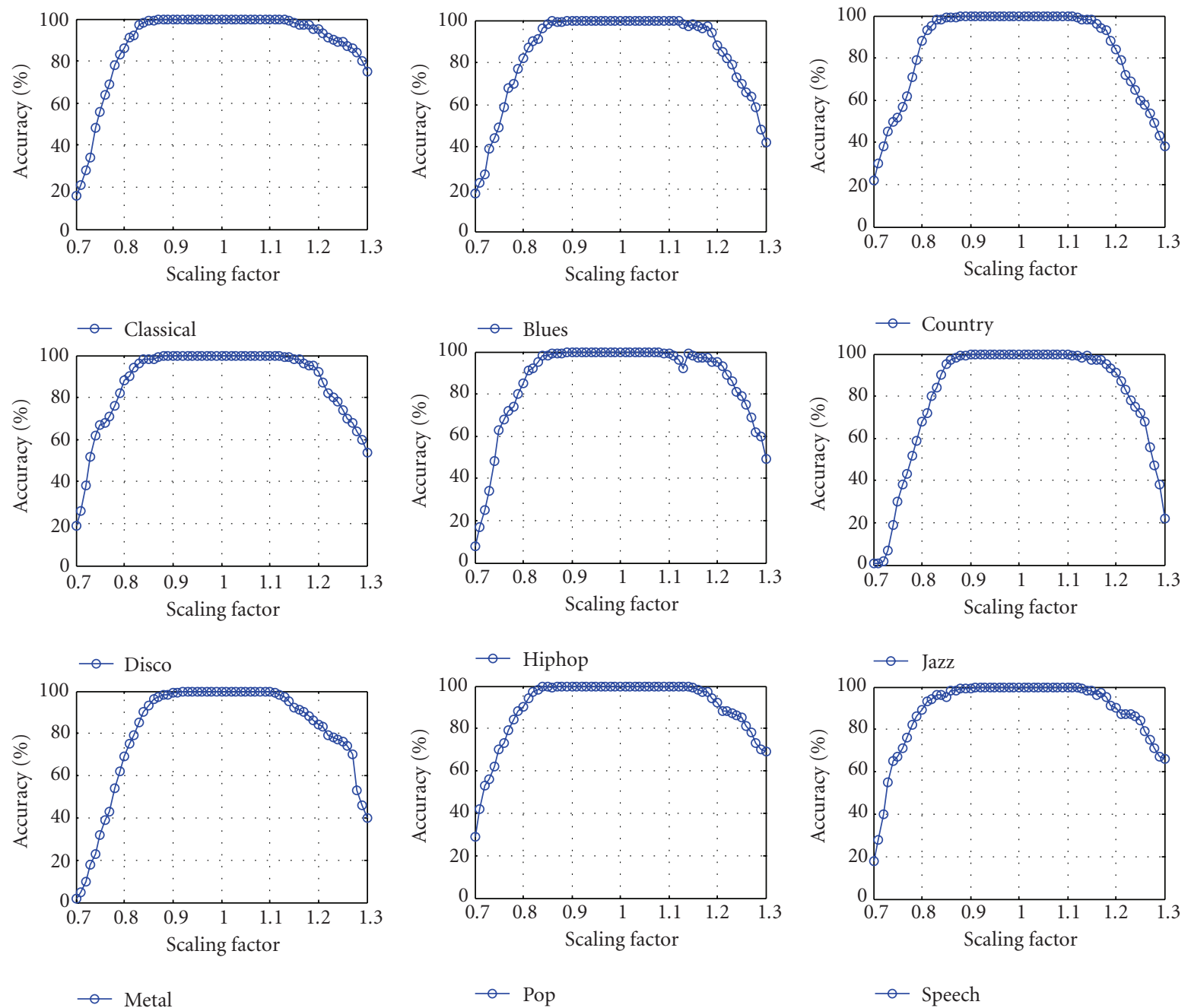

FIGURE 10: Robustness against linear TSM.

Another example is given as follows:

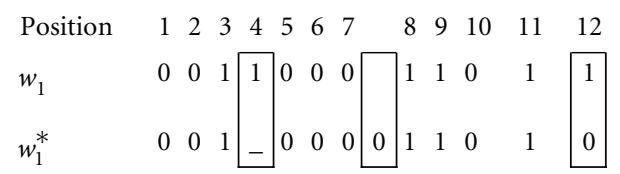

We can see that a deletion error, an insertion error and a substitution error occur at position 4 , between 7 and 8 and at position 12 , respectively.

Due to IDS errors, $w_{1}^{*}$ at the extraction end may take a very different form from $w_{1}$. Traditional ECC schemes, such as BCH coding [10], is not appropriate for IDS channels because they have no ability to resynchronize bits. Some efforts have been made in order to solve this problem. For example, low density parity coding (LDPC) is used to resynchronize the message $[28,29]$. But prior possibilities are needed in these schemes, which are not possible in watermarking applications. In [12], ECC based on repetition coding and HDB3 are proposed to tackle bit desynchronization. However, because of the sensitivity of
HDB3, error propagation may occur during the decoding process which would damage all the trailing watermark bits. In [14], another ECC based on repetition coding is proposed. However, if approximate alignment is not achieved, error propagation would also damage all the trailing watermark bits.

In this paper we carefully design an ECC scheme called repetition-header coding (RHC), with great ability to resynchronize bits. The experimental results show that it has very good robustness against IDS channels.

The original binary watermark $w$ first goes through repetition coding. Then a header is repeatedly inserted into the repetition codes to obtain the encoded watermark $w_{1}$. The structure of the encoded watermark $w_{1}$ is illustrated in Figure 6.

The headers are used to indicate the boundaries of the repetition codes and prevent error propagation in the decoding process. It must have a different form from the repetition code. The header we use here takes the interlaced form 10101010 ...10. 

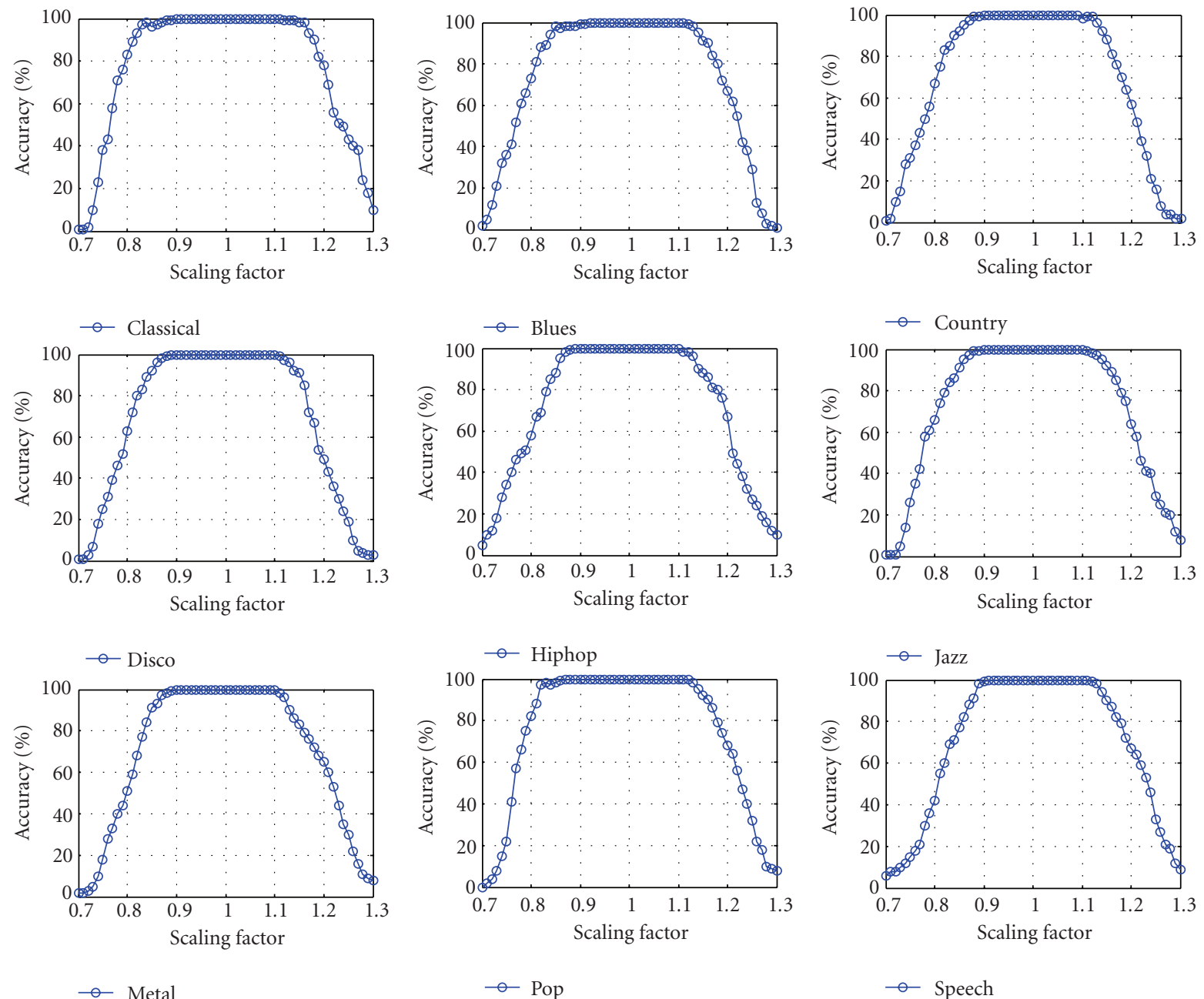

FIGURE 11: Robustness against pitch-invariant TSM.

TABle 1: Distortion.

\begin{tabular}{lcccccccc}
\hline & Classical & Blues & Country & Hiphop & Jazz & Metal & Pop & Speech \\
\hline Average SNR $(\mathrm{db})$ & 18.2 & 20.1 & 21.1 & 24.8 & 25.2 & 24.2 & 21.6 & 22.3 \\
Average ODG & -1.04 & -0.65 & -0.62 & -0.31 & -0.11 & -0.33 & -0.57 & -0.48 \\
\hline
\end{tabular}

For example, suppose the original watermark $w$ is 1001 , the repetition time is 20 and the header length is 10 . Then the encoded watermark $w_{1}$ would be:

$$
\begin{aligned}
w= & 1001=> \\
w_{1}= & 1010101010 \underline{11111111111111111111} \\
& 1010101010 \underline{\underline{000000000000000000000}} \\
& 1010101010 \underline{\underline{000000000000000000000}} \\
& 1010101010 \underline{111111111111111111111} \\
& 1010101010
\end{aligned}
$$

The parts underlined are the repetition codes of the original watermark bits. "1010101010" is the header. $w_{1}$ is then embedded into the audio.

The decoding process is as follows.

(1) Differential of $w_{1}^{*}$ to obtain $D(i)$ :

$$
D(i)=w_{1}^{*}(i+1)-w_{1}^{*}(i) .
$$

(2) Bit filtering based on the K Nearest Neighbour Rule (KNNR) is applied on $D(i)$ to obtain $D_{f}(i)$ by (11). According to KNNR, whether a bit is " 1 " or " 0 " depends on its $K$ nearest samples. If more than 
TABle 2: Robustness to Stirmark attacks (Watermark length $=10$ ).

\begin{tabular}{|c|c|c|c|c|c|c|c|c|c|}
\hline Attack & Classical & Blues & Country & Disco & Hiphop & Jazz & Metal & Pop & Speech \\
\hline addbrumm_100 & 0 & 0 & 0 & 0 & 0 & 0 & 0 & 0 & 0 \\
\hline addbrumm_1100 & 0 & 0 & 0 & 0 & 0 & 0 & 0 & 0 & 0 \\
\hline addbrumm_2100 & 0 & 0 & 0 & 0 & 0 & 0 & 0 & 0 & 0 \\
\hline addbrumm_3100 & 0 & 0 & 0 & 0 & 0 & 0 & 0 & 0 & 0 \\
\hline addbrumm_4100 & 0 & 0 & 0 & 0 & 0 & 0 & 0 & 0 & 0 \\
\hline addbrumm_5100 & 0 & 0 & 0 & 0 & 0 & 0 & 0 & 0 & 0 \\
\hline addbrumm_6100 & 0 & 0 & 0 & 0 & 0 & 0 & 0 & 0 & 0 \\
\hline addbrumm_7100 & 0 & 0 & 0 & 0 & 0 & 0 & 0 & 0 & 0 \\
\hline addbrumm_8100 & 0 & 0 & 0 & 0 & 0 & 0 & 0 & 0 & 0 \\
\hline addbrumm_9100 & 0 & 0 & 0 & 0 & 0 & 0 & 0 & 0 & 0 \\
\hline addbrumm_10100 & 0 & 0 & 0 & 0 & 0 & 0 & 0 & 0 & 0 \\
\hline addfftnoise & - & - & - & - & - & - & - & - & - \\
\hline addnoise_100 & 0 & 0 & 0 & 0 & 0 & 0 & 0 & 0 & 0 \\
\hline addnoise_300 & 0 & 0 & 0 & 0 & 0 & 0 & 0 & 0 & 0 \\
\hline addnoise_500 & 0 & 0 & 0 & 0 & 0 & 0 & 0 & 0 & 0 \\
\hline addnoise_700 & 0 & 0 & 0 & 0 & 0 & 0 & 0 & 0 & 0 \\
\hline addnoise_900 & 0 & 0 & 0 & 0 & 0 & 0 & 0 & 0 & 0 \\
\hline addsinus & 0 & 0 & 0 & 0 & 0 & 0 & 0 & 0 & 0 \\
\hline amplify & 0 & 0 & 0 & 0 & 0 & 0 & 0 & 0 & 0 \\
\hline compressor & 0 & 0 & 0 & 0 & 0 & 0 & 0 & 0 & 0 \\
\hline copysample & 0 & 0 & - & 0 & 0 & 0 & - & 0 & 0 \\
\hline cutsamples & 0 & 0 & 0 & 0 & 0 & 0 & 0 & 0 & 0 \\
\hline dynnoise & 0 & 0 & 0 & 0 & 0 & 0 & 0 & 0 & 0 \\
\hline echo & - & - & - & - & - & - & - & - & - \\
\hline exchange & 0 & 0 & 0 & 0 & 0 & 0 & 0 & 0 & 0 \\
\hline extrastereo_30 & 0 & 0 & 0 & 0 & 0 & 0 & 0 & 0 & 0 \\
\hline extrastereo_50 & 0 & 0 & 0 & 0 & 0 & 0 & 0 & 0 & 0 \\
\hline extrastereo_70 & 0 & 0 & 0 & 0 & 0 & 0 & 0 & 0 & 0 \\
\hline fft_hlpass & 0 & 0 & 0 & 0 & 0 & 0 & 0 & 0 & 0 \\
\hline fft_invert & - & - & - & - & - & - & - & - & - \\
\hline fft_real_reverse & 0 & 0 & 0 & 0 & 0 & 0 & 0 & 0 & 0 \\
\hline fft_stat1 & - & - & - & & - & - & - & - & - \\
\hline fft_test & - & - & - & & - & - & - & - & - \\
\hline flippsample & 0 & - & 0 & 0 & 0 & 0 & - & 0 & 0 \\
\hline invert & - & - & - & - & - & - & - & - & - \\
\hline lsbzero & 0 & 0 & 0 & 0 & 0 & 0 & 0 & 0 & 0 \\
\hline normalize & 0 & 0 & 0 & 0 & 0 & 0 & 0 & 0 & 0 \\
\hline nothing & 0 & 0 & 0 & 0 & 0 & 0 & 0 & 0 & 0 \\
\hline original & 0 & 0 & 0 & 0 & 0 & 0 & 0 & 0 & 0 \\
\hline rc_highpass & 0 & 0 & 0 & 0 & 0 & 0 & 0 & 0 & 0 \\
\hline rc_lowpass & 0 & 0 & 0 & 0 & 0 & 0 & 0 & 0 & 0 \\
\hline smooth & 0 & 0 & 0 & 0 & 0 & 0 & 0 & 0 & 0 \\
\hline smooth2 & 0 & 0 & 0 & 0 & 0 & 0 & 0 & 0 & 0 \\
\hline stat1 & 0 & 0 & 0 & 0 & 0 & 0 & 0 & 0 & 0 \\
\hline stat2 & 0 & 0 & 0 & 0 & 0 & 0 & 0 & 0 & 0 \\
\hline voiceremove & - & - & - & - & - & - & - & - & - \\
\hline zerocross & 0 & 0 & 0 & 0 & 0 & 0 & 0 & 0 & 0 \\
\hline zerolength & 0 & 0 & 0 & 0 & 0 & 0 & 0 & 0 & 0 \\
\hline zeroremove & 0 & 0 & 0 & 0 & 0 & 0 & 0 & 0 & 0 \\
\hline
\end{tabular}



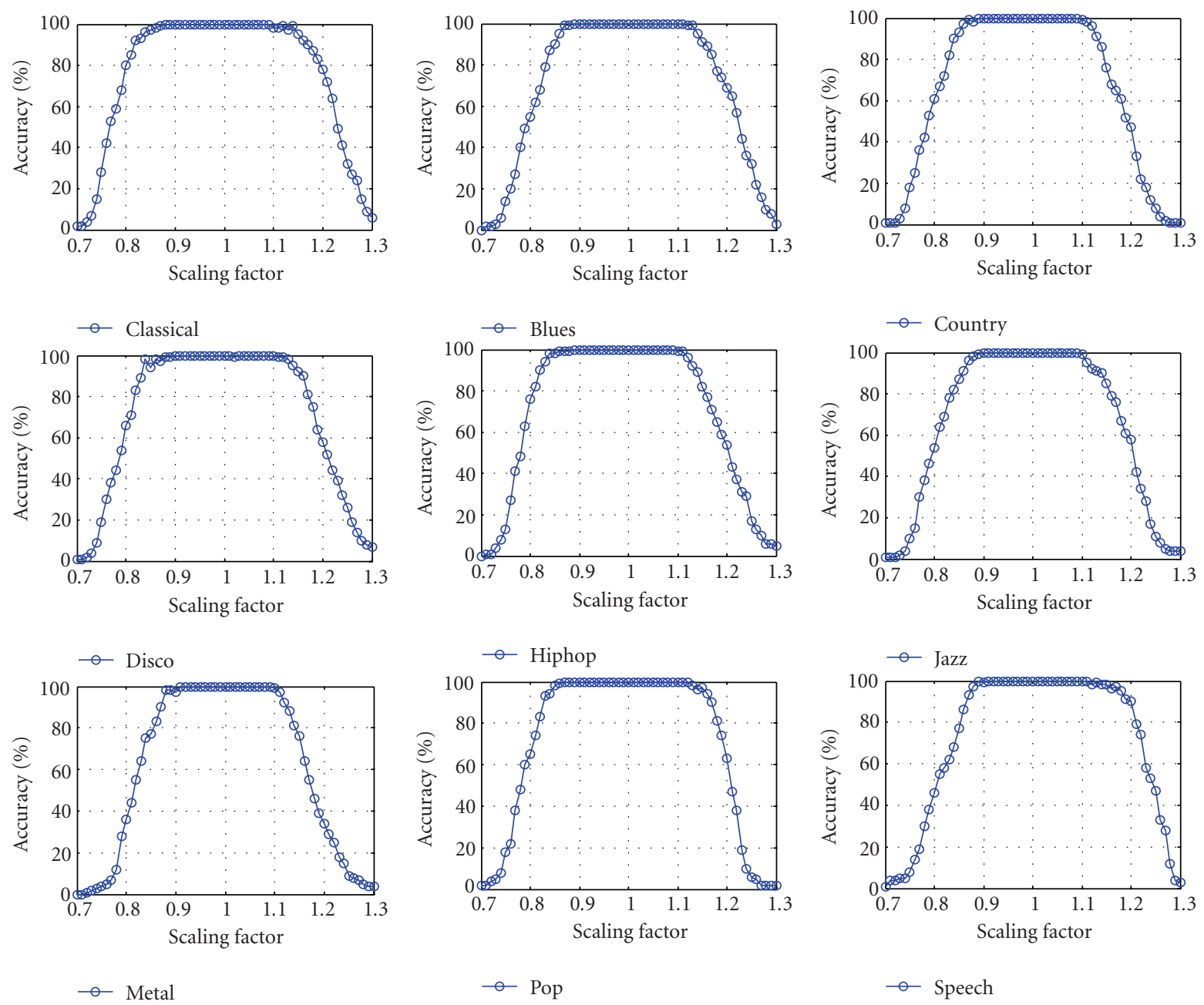

FIGURE 12: Robustness against PSM.

TABLE 3: Robustness to cropping (Watermark length $=10$ ).

\begin{tabular}{lccc}
\hline Time cropped & Result & Time cropped & Result \\
\hline $1 \%$ & 1010011010 & $6 \%$ & $101001 \_010$ \\
$2 \%$ & 1010111010 & $7 \%$ & $101001 \_010$ \\
$3 \%$ & $101011 \_010$ & $8 \%$ & $101001 \_010$ \\
$4 \%$ & $101001 \_010$ & $9 \%$ & $101001 \_010$ \\
$5 \%$ & $101001 \_010$ & $10 \%$ & $101001 \_010$ \\
\hline
\end{tabular}

$K / 2$ samples are " 1 ", this bit is considered as " 1 "; otherwise it is considered as " 0 ", as illustrated in (10) and Figure 7 (Here we use the letter $r$ instead of $K ; r$ is called the filtering diameter).

$$
D_{f}(i)= \begin{cases}1 & \text { if }(t>z) \\ 0 & \text { if }(t \leq z),\end{cases}
$$

where $z=\lfloor r / 2\rfloor$, and $t=\sum_{j=i-z}^{j=i+z} D(j)$.
(3) Suppose the starting and ending position of the $i$ th consecutive " 0 " sequence in $D_{f}$ are pStart and $p E n d$, respectively. Then $w^{*}(i)$ is extracted from $w_{1}^{*}$ (pStart : $\left.p E n d\right)$ according to (12). $L_{i}$ is the length of this " 0 " sequence and $t$ is the number of bits " 1 " in $w_{1}^{*}(p$ Start $:$ pEnd $)$.

$$
w^{*}(i)= \begin{cases}1 & \text { if }\left(t>\left\lfloor\frac{L_{i}}{2}\right\rfloor\right) \\ 0 & \text { if }\left(t \leq\left\lfloor\frac{L_{i}}{2}\right\rfloor\right) .\end{cases}
$$

Let us take the above $w=1001$ as an example. The repetition time is 20 and the header length is 10. Suppose the watermarked audio is modified during transmission and some IDS errors occur. Then the extracted watermark $w_{1}^{*}$ will be different from $w_{1}$. Let us suppose it to be (substitution 

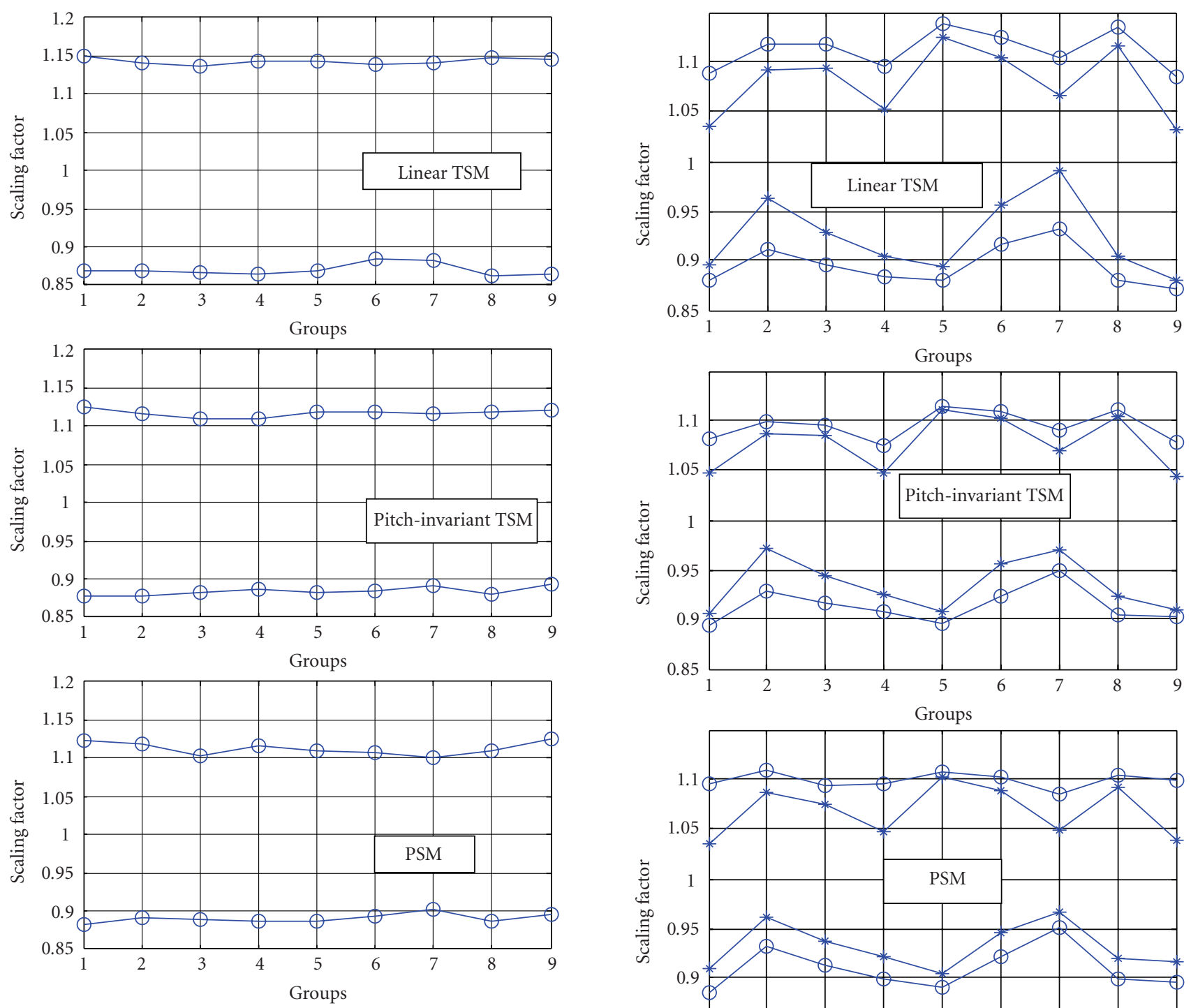

Figure 13: Average upper and lower bounds, watermark length = 10.

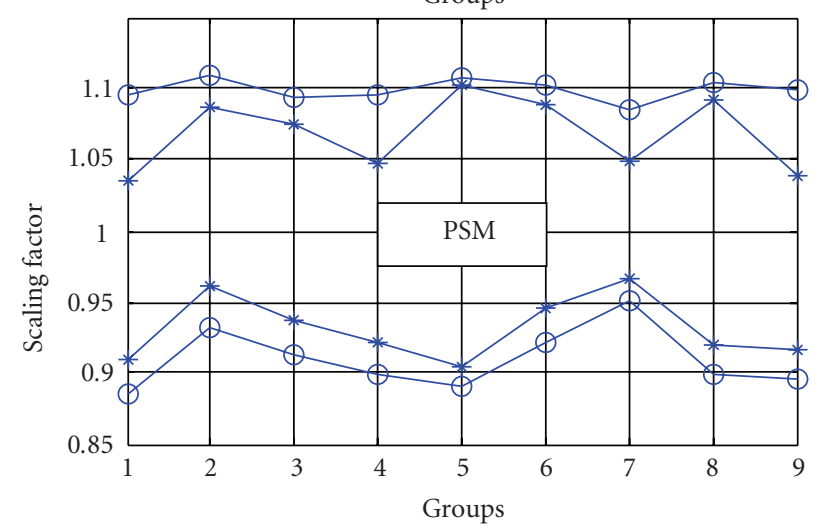

FIGURE 14: Average upper and lower bounds. o: Watermark length $=20,{ }^{*}$ : Watermark length $=40$.

or insertion errors marked by bold and deletion errors marked by underlines)

$$
\begin{aligned}
& w_{1}^{*}=1010111010111111111011111011111 \\
& 1 \underline{0010101000000110000000000000 \quad 010101010} \\
& 0001000000000000000010101010010
\end{aligned}
$$$$
11111111100111111 \underline{1} 1010101010
$$

The decoding process is as follows.

(1) Calculate $D(i)$ according to (10). We get

$$
\begin{aligned}
D(i)= & 1111 \underline{0} 0111100000000110000110000 \\
& 010111111000001010000000000000 \\
& 111111110001100000000000000 \\
& 11111111011100000000101000000 \\
& 0111111111
\end{aligned}
$$

(2) Bit filtering. Here suppose $r=8$. Let us take the underlined $D(5)=0$ for example. According to (11), $t=7>z=4$ which means that $D_{f}(5)=1$. We can see that this wrong bit has been corrected by its 


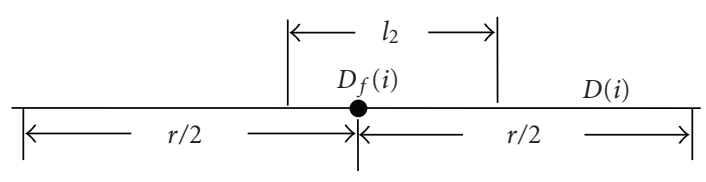

FIGURE 15: Bit filtering when $l_{2}<r / 2$.

nearby correct bits. Then we obtain:

$$
D_{f}=11111111 \underline{0} \underline{0} 00000000000000000000
$$

$00 \underline{0} 111111 \underline{000000000000000000000 ~}$

$11111111 \underline{0} 000000000000000000$

\section{0}

\section{1}

We see that the consecutive " 1 " sequences indicate the approximate positions of the header and the consecutive " 0 " sequences indicate the approximate positions of the repetition codes.

(3) The starting and ending position (the underlined bits) of the 1st 0 -sequence is 10 and 33, respectively, in the above $D_{f}$. So $L_{1}=24$. The corresponding segment in $w_{1}^{*}$ is $w_{1}^{*}(10: 33)$ : 011111111101111101111110. Then here $t=20>$ $L_{1} / 2=12$. According to $(12), w^{*}(1)=1$. The same rule is applied to the rest of the sequences and we obtain $w^{*}=1001$, which is identical to the original watermark $w$.

Here we present an experimental result in Figure 8. $w=$ $\left[\begin{array}{llll}1 & 0 & 1 & 0\end{array}\right]$. Repetition time $l_{1}=400$ and header length $l_{2}=200$. After embedding, a linear TSM (scale factor 1.1) is applied to the watermarked clip. From Figure 8 we can see that $w_{1}^{*}$ is quite different from $w_{1}$ due to the IDS errors. And $D$ is so noisy that it is impossible to distinguish the boundaries of the repetition codes. However, $D_{f}$, the bitfiltered version of $D$, very clearly indicates the locations of the repetition codes (compare $D_{f}$ with $w_{1}$ ), and thus it can be used to recover the final watermark $w^{*}$ from $w_{1}^{*}$.

There exists a problem with choosing the values for the repetition time $l_{1}$, the header length $l_{2}$ and the filtering diameter $r$. We outline a model for these parameters in Appendix B. In our algorithm $r=l_{2}$ and $l_{2}=l_{1} / 2$ have the best performance according to experiments.

\section{Experimental Results}

In the experiments, we tested 900 audio clips. They are divided into nine groups: classical, blues, country, disco, hiphop, jazz, metal, pop, and speech, numbered as groups 1 to 9. Each group consists of 100 clips. The group of classical clips consists of various musical instruments. The group of speech consists of news reports and dialogues. Other groups consist of different human voices with different backgrounds for entertainment. All clips are of wav format, $44.1 \mathrm{k}$ sampling rate, 16-bit quantization, mono. db2 is selected as the wavelet basis. The wavelet decomposition level is 3 for classical and 5 for the other groups. The program is run in Matlab 7. The attacks we consider here are temporally linear TSM, pitch-invariant TSM and PSM, along with others such as cropping, jittering, MP3, resampling, requantization, and Stirmark for Audio.

4.1. Embedding Distortion. In the embedding process, the distortion relies on the widths of Shape-A and Shape-B, the number of bits in $w_{1}$ and the DYWT decomposition level. The widths of the two shapes are the embedding strength. The larger the widths, the more samples are modified and the more robust will be the watermark with greater distortion. The number of bits in $w_{1}$ is the product of the original watermark length and $\left(l_{1}+l_{2}\right)$. The larger $l_{1}$, and $l_{2}$ are, the greater the robustness and distortion will be. Therefore we can adjust the above parameters to an acceptable balance between distortion and robustness. In the experiments we embedded a watermark of 10 bits, and adopted $l_{1}=200$ and $l_{2}=100$ when the distortion and robustness are balanced.

In the proposed algorithm, the embedded bits are located by the peak widths and the bit decision is made according to the peak height. Therefore the performance of the algorithm is based on the fluctuations of the embedding domain. If the embedding domain is too flat, it will be difficult to embed the watermark bits; the robustness will be weak as well. From Figure 9(a) we can see that as the decomposition level grows, the waveform of DYWT coefficients becomes flatter and flatter. So the decomposition level should not be too large. On the other hand, the degradation of quality is affected by the decomposition level. The smaller the decomposition level, the greater the distortion will be. So the decomposition level should not be too small. As a result there is a trade-off. In the experiments, we found that a decomposition level of 5 was acceptable for most of the audio clips when robustness, distortion and capacity were taken into account. But for the classical group, the robustness was not as good as other groups. The reason lies in the fact that the waveform of 5 level decomposition coefficients is flatter than the other groups as shown in Figure 9(b) where we intercept and present a short segment of the 5 level DYWT decomposition coefficients from a classical and a jazz clip, respectively. Therefore, for the classical group, we reduced the decomposition level to 3 so that a good robustness was achieved.

We tested the distortion with values of SNR and objective difference grade (ODG). The SNR value can reflect the degree of modification brought by the watermarking while the ODG value reflects the human auditory system (HAS) model to show the distorted degree of audio frames. According to the requirement of the International Federation of the Phonographic Industry (IFPI), the SNR value should be higher than $20 \mathrm{~dB}$ [20]. The ODG value can be mapped to the following description [30]: 0 (insensitive), -1 (audible), -2 (slightly annoying), -3 (annoying), -4 (very annoying), and -5 (catastrophic). The value of OGD is obtained by EAQUAL 0.1.3 alpha [31]. 
TABLE 4: Robustness to jittering (Watermark length $=10$ ).

\begin{tabular}{lccccccccc}
\hline Jittering & classical & blues & Country & disco & hiphop & jazz & metal & pop & speech \\
\hline$-(1: 15)$ & 0 & 0 & 0 & 0 & 0 & 0 & 0 & 0 & 0 \\
$-(1: 11$ & 0 & 0 & 0 & 0 & 0 & 0 & 0 & 0 & 0 \\
$-(1: 10)$ & - & 0 & 0 & - & 0 & 0 & 0 & - & 0 \\
$-(1: 8)$ & - & - & - & - & 0 & - & 0 & - & - \\
$-(1: 5)$ & - & - & - & - & - & - & - & - & - \\
$+(1: 15)$ & 0 & 0 & 0 & 0 & 0 & 0 & 0 & 0 & 0 \\
$+(1: 11)$ & 0 & 0 & 0 & 0 & 0 & 0 & 0 & 0 & 0 \\
$+(1: 10)$ & 0 & 0 & 0 & 0 & 0 & 0 & 0 & 0 & 0 \\
$+(1: 8)$ & 0 & 0 & 0 & 0 & 0 & 0 & 0 & 0 & - \\
$+(1: 5)$ & - & - & - & - & 0 & - & 0 & - & - \\
\hline
\end{tabular}

From Table 1, we can see that the watermark is imperceptible in most of the clips since most of the SNRs are over $20 \mathrm{db}$ and the ODGs are between $-1 \sim 0$. But for classical, the audio quality shows a slight degradation because the decomposition level for this group is 3 . We can reduce the distortion by reducing the watermark length, the repetition times or the header length. For example, when we embed a shorter watermark ( 5 bits) in the 5 level decomposition DYWT, it becomes inaudible, but, for clarity, we still chose to present the results when all the parameters except the decomposition level were the same.

\subsection{Robustness Test}

4.2.1. Robustness to TSM and PSM. In Figures 10-12 we present the robustness to linear TSM, pitch-invariant TSM and PSM, when the scale factors range from 0.7 to $1.3( \pm 30 \%$ scaling). The vertical axis, accuracy, is the percentage of clips from which $w^{*}$ is the same as $w$. Since each group consists of 100 clips, $N \%$ means the watermark can be completely recovered from $N$ audio clips without any errors in this group. From Figure 10, we see that in the classical group, when the linear TSM scale factors are within $[0.85$, 1.15] the accuracy is almost $100 \%$, that is the watermark is completely recovered from almost all of the clips. And when the scale factors are within $[0.8,1.26]$ the accuracy is more than $80 \%$, meaning that the watermark survives in more than $80 \%$ of the clips. We can also see that for different audio clips, the degree of tolerance of the watermark is different. For example, in the classical group, for some audio clips the watermark can resist 0.7 or 1.3 scaling; but for some others only around 0.85 or 1.15 at most. For one clip, if the watermark can survive within $\left[N_{1}, N_{2}\right]$, $N_{1}$ and $N_{2}$ are called the lower bound and upper bound, respectively. We compute the average lower bound and upper bound for each group, and present them in Figure 13. We see that, statistically, the watermark can resist scaling within $[0.87,1.16]([-13 \%,+16 \%])$ in the classical group. For other groups, the average lower bounds are between 0.86 and 0.9 , and the average upper bounds are around 1.16. The average scaling tolerance for linear TSM is around $[0.88,1.16]([-12 \%,+16 \%])$ statistically, which means that our algorithm is robust to linear TSM.
Similar results are shown in Figures 11 and 12 with respect to pitch-invariant TSM and PSM. From Figure 13, we see that, statistically, the watermark can resist pitchinvariant TSM of around $[0.86,1.12]([-14 \%,+12 \%])$ and PSM of around $[0.88,1.12]([-12 \%,+12 \%])$, which means that our algorithm is also robust against these two kinds of scaling.

The robustness depends on the similarity of Set A and Set B and on the RHC scheme. We observed one clip in the speech group where the temporally linear TSM reaches 0.82 $(-18 \%)$ and found that the intersection of Set A and Set B is as high as around $60 \%$. That is around $60 \%$ bits of $w_{1}^{*}$ are extracted from the watermarked peaks; the other $40 \%$ bits are extracted from the un-watermarked peaks. Many IDS errors occur during scaling. But our specially designed RHC decoder successfully fixes these desynchronization errors and recovers the original watermark. However when the scaling reaches $0.8(-20 \%)$, the intersection of Set A and Set B is only around $40 \%$. The IDS errors are too serious to erase and the RHC decoder cannot recover the correct watermark from $w_{1}^{*}$.

In conclusion, our algorithm is robust against both TSM and PSM. On the contrary no other scheme reported so far in the literature can overcome both TSM and PSM, as stated in Section 2. For example, $[16,20]$ can only deal with TSM, but are unable to overcome PSM. In this paper, we solve this problem by the invariant features of DYWT, the robust embedded methods and the RHC scheme.

In the above scaling, the original watermark contains 10 bits, $l_{1}=200$ and $l_{2}=100$. We now change these values: (1) watermark length $=20, l_{1}=100$ and $l_{2}=50$; (2) watermark length $=40, l_{1}=50$ and $l_{2}=25$. The average upper and lower bounds are shown in Figure 13. Statistically, the average tolerances for the nine groups of linear TSM, pitch-invariant TSM and PSM are [0.895, 1.112], [0.914, $1.095],[0.910,1.099]$ (watermark length $=20$ ) and [0.924, $1.08],[0.935,1.078],[0.932,1.069]$ (watermark length $=$ 40). That is, the watermark can resist scaling of about $\pm 10 \%$ when the watermark capacity is 2 bps and about $\pm 8 \%$ when the capacity is $4 \mathrm{bps}$. Although, statistically, the robustness is good, the watermark may be fragile in some particular audio clips. For example, from Figure 14, we see that the robustness is not so good in group 7 (metal). How to integrate the 
TABLE 5: Comparison with other algorithms.

\begin{tabular}{|c|c|c|c|c|}
\hline Algorithm & Capacity & Robustness to TSM & Robustness to PSM & Robustness to other attacks \\
\hline$[12]$ & $2.3 \mathrm{bps}$ & Around $\pm 8 \%$ & Unreported & Unreported \\
\hline$[16]$ & $4.2 \mathrm{bps}$ & $\begin{array}{l}\text { Pitch-invariant TSM of around } \\
\pm 7 \% \text {, but susceptible to linear } \\
\text { TSM }\end{array}$ & Susceptible to PSM & Unreported \\
\hline [20] & 3 bps & $\begin{array}{l}\text { Pitch-invariant TSM of around } \\
\pm 20 \% \text {, and linear TSM of } \\
\text { around } \pm 10 \%\end{array}$ & Susceptible to PSM & $\begin{array}{l}\text { Susceptible to MP3 compression: } \\
\text { errors occur under the smallest } \\
\text { compression ratio. }\end{array}$ \\
\hline [23] & Unreported & Susceptible to TSM & $\begin{array}{l}\text { BER is around } 13 \% \text { when scaling } \\
\text { factors are } \pm 10 \%\end{array}$ & $\begin{array}{l}\text { Robust to compression ratio of } \\
11 .\end{array}$ \\
\hline Ours & $1 \mathrm{bps}-4 \mathrm{bps}$ & $\begin{array}{l}\text { Pitch-invariant TSM of around } \\
{[-14 \%,+12 \%], \text { and linear TSM }} \\
\text { of around }[-12 \%,+16 \%] \text { at a } \\
\text { capacity of } 1 \mathrm{bps} ; \pm 10 \% \text { and } \pm 8 \% \\
\text { at } 2 \text { bps and } 4 \mathrm{bps}, \text { respectively. }\end{array}$ & $\begin{array}{l}\text { PSM of around }[-12 \%,+12 \%] \\
\text { at a capacity of } 1 \mathrm{bps} ; \pm 10 \% \text { and } \\
\pm 8 \% \text { at } 2 \text { bps and } 4 \text { bps, } \\
\text { respectively. }\end{array}$ & $\begin{array}{l}\text { Very robust to MP3 compression } \\
\text { even under the largest } \\
\text { compression ratio } 22.05 \text {. }\end{array}$ \\
\hline
\end{tabular}

different properties of the audio signals into the algorithm is a major challenge in the future.

4.2.2. Robustness against Stirmark for Audio. Stirmark for Audio is a benchmark software in audio watermarking. We present the results for one clip from each group in Table 2. " 0 " denotes that $w^{*}$ is the same as $w$. And "—" denotes that $w^{*}$ is different from $w$. We can see that the watermark is robust against most attacks. But the watermarked audio signal is made very noisy by some attacks, such as "fft_noise", "fft_stat1", "fft_test", "echo" and "voicerremove". The SNR values after these attacks all drop to $2 \sim 4 \mathrm{db}$, which means that the attacked audio is totally destroyed and it is reasonable that the watermark is erased. "invert" attack also destroys the watermark because it inverts the whole waveform of the signal so that the peaks become the troughs and the troughs become the peaks. As the watermarked peaks are changed into troughs by the "invert" attack, the watermark becomes undetectable if we search the peaks for embedded bits. However, this is not a big problem. The solution is to search the troughs if we fail to extract the watermark from the peaks or to revise the embedding algorithm so that the watermark bits are embedded into the widest peaks and troughs.

4.2.3. Robustness to Cropping and Jittering. Now randomly selected portions of the clips are cropped. As the results for all clips are similar, we show in Table 3 the results for a symphony clip from the classical group. It can resist $2 \%$ cropping. By comparing with the original watermark 1010011010, we can see that the error propagation is restricted, that is, lost watermark bits are limited within the cropped portion, but the other watermark bits in the remaining parts are not affected. If the cropped portion contains no watermark bits, the watermark can be completely recovered.

We also performed jittering on the clips and show the results for one clip from each group in Table 4, from which we can see that the watermark is robust to jittering of around $\pm(1: 10)$. That is, when 1 sample is copied into or cut off from every 10 samples the watermark still survives. Compared with [20], in which the watermark can resist \pm (1 : $50)$ jittering, our algorithm has a much better performance.

4.2.4. Robustness against Other Common Operations. MP3 compression ratios are 5.5 (128 kbps), 7.4 (96 kbps), 8.8 (80 kbps), 11.0 (64 kbps), 12.6 (56 kbps), 14.7 (48 kbps), 17.64 (40 kbps), 22.05 (32 kbps). The watermark (10 bits) cannot survive from 45 clips, $5 \%$ of the 900 clips, when the compression ratio is 22.05 . The watermark survives in all clips subjected to re-sampling $(44.1 \mathrm{k} \rightarrow 11.025 \mathrm{k} \rightarrow$ $44.1 \mathrm{k}$ ) and re-quantization (16 bits $\rightarrow 8$ bits $\rightarrow 16$ bits). The watermark is very robust against these operations because, basically, MP3, re-sampling and requantization cause little geometrical desynchronization and they do not pose big challenges to audio watermarking.

4.3. Comparison with Other Reported Efforts. From Table 5, we can see that other algorithms cannot resist TSM or PSM because the domains for data embedding do not have invariant properties. For example, in [16], the data is embedded in the FFT domain of the DWT coefficients. If the frequency components are changed by PSM modifications, the watermark would be lost. In [20], data is embedded by modifying the relationship between the sample values in the time domain. However PSM would seriously damage such a relationship. In [23], the extraction process is based on the assumption that the time duration remains unchanged. So when the time duration is changed by TSM or tempovariant PSM, the watermark would become undetectable. In our algorithm, the DYWT has invariant properties both to TSM and PSM, which help retain the watermark.

\section{Discussion and Conclusion}

In summary, an audio watermarking algorithm based on the DYWT and an HRC coding scheme robust against geometrical distortions and other common operations is 
proposed in this paper. The main contributions are listed as follows.

(1) The DYWT is examined thoroughly by theoretical deduction and extensive experiments. Based on the analysis, we conclude that the DYWT has very good geometrical invariance compared with DWT, DCT and DFT.

(2) Resynchronization is achieved by utilizing the geometrical invariance of the DYWT. The widest peaks of the DYWT coefficients are selected to embed the watermark bits. At the receiving end, the widest peaks are considered as containing the watermark bits. Experimental results show that this is an effective way to identify the watermarked positions. A novel embedding method using two different waveforms to represent the bits "0" and "1" is proposed. Blind detection is realized.

(3) We also design a special ECC scheme called RHC that significantly helps to recover the watermark and restricts error propagation due to IDS errors.

(4) The proposed algorithm is very robust against desynchronization attacks such as TSM, PSM, jittering, cropping, other common audio processing and Stirmark for Audio. It also has the best performance compared with other reported efforts as shown in Table 5.

However, there exist some issues that must be noted and solved in the future.

(1) In Appendix B, the analysis of (B.1)-(B.3) is based on the deletion error only, regardless of insertion and deletion. In future work a more sophisticated model of IDS errors will be formulated.

(2) The values of parameters such as $r, l_{1}, l_{2}$ and the decomposition level are not determined adaptively. In future work, the algorithm will be refined so that these values can be chosen adaptively. The solution is to integrate the properties of different kinds of audio signal into the algorithm so that these values can balance the distortion and robustness

\section{Appendices}

\section{A.}

We prove the time-shift properties of DWT as follows:

If the steps along the vertical axis and the time axis are $a_{0}$ and $\tau_{0}$, the DWT wavelet can be expressed as

$$
\psi_{j, k}(t)=a_{0}^{-j / 2} \psi\left(a_{0}^{-j} t-k \tau_{0}\right) .
$$

Suppose $\mathrm{WT}_{f}(j, k)$ are the DWT coefficients of $f(t)$. Then

$$
\begin{aligned}
\mathrm{WT}_{f}(j, k) & =\int f(t) \psi_{j, k}^{*}(t) d t \\
& =\int f(t) \cdot a_{0}^{j / 2} \psi^{*}\left(a_{0}^{-j} t-k \tau_{0}\right) d t .
\end{aligned}
$$

Let $f^{\prime}=f\left(t-t_{0}\right)$, then

$$
\begin{aligned}
\mathrm{WT}_{f^{\prime}}(j, k) & =\int f\left(t-t_{0}\right) \psi_{j, k}^{*}(t) d t \\
& =\int f(t) \cdot a_{0}^{j / 2} \psi^{*}\left(a_{0}^{-j} t+a_{0}^{-j} t_{0}-k \tau_{0}\right) d t
\end{aligned}
$$

If

$$
t_{0}=n a_{0}^{j} \tau_{0}
$$

then

$$
\begin{aligned}
\mathrm{WT}_{f^{\prime}}(j, k) & =\int f(t) \cdot a_{0}^{j / 2} \psi^{*}\left(a_{0}^{-j} t+a_{0}^{-j} t_{0}-k \tau_{0}\right) d t \\
& =\int f(t) \cdot a_{0}^{j / 2} \psi^{*}\left(a_{0}^{-j} t-(k-n) \tau_{0}\right) d t \\
& =\mathrm{WT}_{f}(j, k-n) .
\end{aligned}
$$

We can see from (A.5) that, when condition (A.4) holds, the $j$ th level DWT coefficients will be shifted $n$ positions. We also know that for an audio signal of sampling frequency $F$ to be formed by the DWT, $\tau_{0}=1 / F$. Suppose that $t_{0}=N / F$, that is, the audio signal is shifted $N$ positions, then from condition (A.4) we get

$$
N=n \cdot a_{0}^{j} .
$$

From the above, we can show that if the audio signal is shifted $N$ positions and $N$ meets condition (A.6), its $j$ th level DWT coefficients will be shifted $n$ positions in the same direction. In the case of a two filter DWT, $a_{0}=2$. Condition (A.6) is then as follows:

$$
N=n \cdot 2^{j}
$$

\section{B.}

Regardless of insertion and deletion errors, and in connection with substitution errors only, we suppose the probability of a substitution error in every bit to be $p$ and that one bit is independent of another. $X$ is a random variable that stands for the number of erroneous bits in $w_{1}^{*}$ before the KNNR decision. Then we obtain

$$
P\{X=k\}=C_{n}^{k} p^{k}(1-p)^{n-k} .
$$

Then the probability of a correct decision is

$$
\begin{aligned}
P\left\{X<\frac{r}{2}\right\} & =\sum_{k=0}^{r / 2-1} P\{X=k\} \\
& =\sum_{k=0}^{r / 2-1} C_{n}^{k} p^{k}(1-p)^{n-k},
\end{aligned}
$$


We now suppose that $\lambda=r \cdot p$ is a constant and $r \geq 20$. From the Poisson Theory, we further obtain

$$
P\left\{X<\frac{r}{2}\right\} \approx \sum_{k=0}^{r / 2-1} \frac{\lambda^{k} e^{-\lambda}}{k !} .
$$

Let $\lambda=5$. If $r \geq 27, P\{X<r / 2\}$ will be larger than $1-10^{-2}$; if $r \geq 41, P\{X<r / 2\}$ will be larger than $1-10^{-6}$.

Moreover $l_{2} \geq r / 2$ must hold. Otherwise, the consecutive "0" sequence in $D$ may probably be eliminated because part of the filtering window outside the 0 -sequence is longer than the part inside it, as illustrated in Figure 15.

\section{References}

[1] J. Dittmann, A. Mukherjee, and M. Steinebach, "Mediaindependent watermarking classification and the need for combining digital video and audio watermarking for media authentication," in Proceedings of the International Conference on Information Technology: Coding and Computing, pp. 62-67, Las Vegas, Nev, USA, 2002.

[2] F. Deguillaume, S. Voloshynovskiy, and T. Pun, "Method for the estimation and recovering from general affine transforms in digital watermarking applications," in Security and Watermarking of Multimedia Contents IV, vol. 4675 of Proceedings of SPIE, pp. 313-322, San Jose, Calif, USA, 2002.

[3] J. J. K. O'Ruanaidh and T. Pun, "Rotation, scale and translation invariant spread spectrum digital image watermarking," Signal Processing, vol. 66, no. 3, pp. 303-317, 1998.

[4] G. W. Braudaway and F. Mintzer, "Automatic recovery of invisible image watermarks from geometrically distorted images," in Security and Watermarking of Multimedia Contents I, vol. 9 of Proceedings of SPIE, pp. 3971-483, 2000.

[5] S. Pereira and T. Pun, "Robust template matching for affine resistant image watermarks," IEEE Transactions on Image Processing, vol. 9, no. 6, pp. 1123-1129, 2000.

[6] C.-Y. Lin, M. Wu, J. A. Bloom, I. J. Cox, M. L. Miller, and Y. M. Lui, "Rotation, scale, and translation resilient watermaking for images," IEEE Transactions on Image Processing, vol. 10, no. 5, pp. 767-782, 2001.

[7] X. Kang, J. Huang, Y. Q. Shi, and Y. Lin, "A DWT-DFT composite watermarking scheme robust to both affine transform and JPEG compression," IEEE Transactions on Circuits and Systems for Video Technology, vol. 13, no. 8, pp. 776-786, 2003.

[8] L. Cai and S. Du, "Rotation, scale and translation invariant image watermarking using Radon transform and Fourier transform," Proceedings of the IEEE 6th Circuits and Systems Symposium on Emerging Technologies: Frontiers of Mobile and Wireless Communication, vol. 1, pp. 281-284, 2004.

[9] Y. Xin, S. Liao, and M. Pawlak, "Geometrically robust image watermarking via pseudo-Zernike moments," in Proceedings of the Canadian Conference on Electrical and Computer Engineering, vol. 2, pp. 939-942, 2004.

[10] J. Huang and Y. Wang, "A blind audio watermarking algorithm with self-synchronization," in Proceedings of the IEEE International Symposium on Circuits and Systems, vol. 3, pp. 627-630, 2002.

[11] M. F. Mansour and A. H. Tewfik, "Data embedding in audio using time-scale modification," IEEE Transactions on Speech and Audio Processing, vol. 13, no. 3, pp. 432-440, 2005.

[12] M. F. Mansour and A. H. Tewfik, "Time-scale invariant audio data embedding," IEEE Transactions on Multimedia, vol. 3, no. 2, pp. 232-241, 2001.
[13] "SDMI Phase II Screening Technology Version 1.0," February 2000, http://www.usenix.org/publications//library/proceedings/sec01/craver.pdf.

[14] Y. Wang, S. Wu, and J. Huang, "Audio watermarking robust to geometrical distortions based on dyadic wavelet transform," in Security, Steganography, and Watermarking of Multimedia Contents IX, vol. 6505 of Proceedings of SPIE, San Jose, Calif, USA, 2007.

[15] W. Li and X. Xue, "Audio watermarking based on music content analysis: robust against time scale modification," in Proceedings of the 2nd International Workshop on Digital Watermarking (IWDW'04), pp. 13-33, Seoul, South Korea, October 2004.

[16] W. Li, X. Xue, and P. Lu, "Localized audio watermarking technique robust against time-scale modification," IEEE Transactions on Multimedia, vol. 8, no. 1, pp. 60-69, 2006.

[17] L. Cui, S. Wang, and T. Sun, "The application of binary image in digital audio watermarking," in Proceedings of the International Conference on Neural Networks and Signal Processing, vol. 2, pp. 1497-1500, 2003.

[18] L. Cui, S. Wang, and T. Sun, "The application of wavelet analysis and audio compression technology in digital audio watermarking," in Proceedings of the International Conference on Neural Networks and Signal Processing, vol. 2, pp. 15331537, 2003.

[19] W. Li, X. Xue, X. Li, and P. Lu, "A novel feature-based robust audio watermarking for copyright protection," in Proceedings of the International Conference on Information Technology: Coding and Computing [Computers and Communications], vol. 2, pp. 554-558, April 2003.

[20] S. Xiang and J. Huang, "Histogram-based audio watermarking against time-scale modifications and cropping attacks," IEEE Transactions on Multimedia, vol. 9, no. 7, pp. 1357-1372, 2007.

[21] X. Wang, W. Qi, and P. Niu, "A new adaptive digital audio watermarking based on support vector regression," IEEE Transactions on Audio, Speech and Language Processing, vol. 15, no. 8, pp. 2270-2277, 2007.

[22] H.-Y. Liu, X. Zheng, and Y. Wang, "DWT-based audio watermarking resistant to desynchronization," in Proceedings of the 27th IEEE International Conference on Computer and Information Technology, pp. 745-748, 2007.

[23] L. Li, J. Hu, and X. Fang, "Spread-spectrum audio watermark robust against pitch-scale modification," in Proceedings of the IEEE International Conference on Multimedia and Expo (ICME '07), pp. 1770-1773, 2007.

[24] S. Wu, J. Huang, D. Huang, and Y. Q. Shi, "Efficiently selfsynchronized audio watermarking for assured audio data transmission," IEEE Transactions on Broadcasting, vol. 51, no. 1, pp. 69-76, 2005.

[25] J. Laroche, "Time and pitch scale modification of audio signals," in Applications of Digital Signal Processing to Audio and Acoustics, M. Kahrs and K. Brandenburg, Eds., Kluwer Academic Publishers, Norwell, Mass, USA, 1998.

[26] R. J. McAulay and T. F. Quatieri, "Speech analysis/synthesis based on a sinusoidal representation," IEEE Transactions on Acoustics, Speech and Signal Processing, vol. 34, no. 4, pp. 744754, 1986.

[27] http://www.psbspeakers.com/audio-topics/The-Frequenciesof-Music.

[28] M. C. Davey and D. J. C. MacKay, "Reliable communication over channels with insertions, deletions, and substitutions," IEEE Transactions on Information Theory, vol. 47, no. 2, pp. 697-698, 2001. 
[29] M. F. Mansour and A. H. Tewfik, "Convolutional codes for channels with substitutions, insertions, and deletions," in Proceedings of the IEEE Global Telecommunications Conference, vol. 2, pp. 1051-1055, 2002.

[30] M. Steinebach, S. Zmudzinski, and T. Bolke, "Audio watermarking and partial encryption," in Security, Steganography, and Watermarking of Multimedia Contents VII, vol. 5681 of Proceedings of SPIE, pp. 779-788, San Jose, Calif, USA, 2005.

[31] http://www.mp3-tech.org/. 